\title{
Multidimensional Hamiltonian for tunneling with position-dependent mass
}

\author{
Antonio Fernández-Ramos, ${ }^{*}$ Zorka Smedarchina, ${ }^{\dagger}$ and Willem Siebrand ${ }^{\dagger}$ \\ Department of Physical Chemistry and Center for Research in Biological Chemistry and Molecular Materials, \\ University of Santiago de Compostela, 15706 Santiago de Compostela, Spain
}

(Received 2 May 2014; published 15 September 2014)

\begin{abstract}
A multidimensional Hamiltonian for tunneling is formulated, based on the mode with imaginary frequency of the transition state as a reaction coordinate. To prepare it for diagonalization, it is transformed into a lowerdimension Hamiltonian by incorporating modes that move faster than the tunneling into a coordinate-dependent kinetic energy operator, for which a Hermitian form is chosen and tested for stability of the eigenvalues. After transformation to a three-dimensional form, which includes two normal modes strongly coupled to the tunneling mode, this Hamiltonian is diagonalized in terms of a basis set of harmonic oscillator functions centered at the transition state. This involves a sparse matrix which is easily partially diagonalized to yield tunneling splittings for the zero-point level and the two fundamental levels of the coupled modes. The method is tested on the well-known benchmark molecule malonaldehyde and a deuterium isotopomer, for which these splittings have been measured. Satisfactory agreement with experiment results is obtained.
\end{abstract}

DOI: 10.1103/PhysRevE.90.033306

PACS number(s): 02.70.-c, 03.65.Xp, 82.20.Wt

\section{INTRODUCTION}

If a molecule has two (or more) identical stable minima, all vibrational levels are correspondingly double (or multiple) valued. The level splitting is hidden in the usual BornOppenheimer analysis of the equilibrium configurations, but shows up in the analogous analysis of the transition state between the minima as a vibrational degree of freedom with an imaginary frequency. If the potential of this imaginary mode is extended to the equilibrium regions, it takes the form of a double-minimum potential, which gives rise to the usual interpretation of the splitting as due to tunneling through a potential-energy barrier whose maximum is the transition state. In practice such tunneling splittings are usually extremely small unless the tunneling involves light particles, in particular, protons or hydrogen atoms, in which case these splittings offer an important window on the mobility of these particles in systems of chemical and biological interest.

Experimentally, the level splittings are observed as a property of the equilibrium configuration, and early attempts to calculate them were based on the vibrational potential of the equilibrium structure, subjected to a perturbation due to the presence of an identical structure nearby. A survey of recent methods for tunneling in complex systems can be found in Ref. [1]. However, it is more logical to start from the vibrational potential of the transition state [2], since this is the configuration of highest symmetry. In the vicinity of the dividing plane passing through this point, the mode with imaginary frequency, which acts as a reaction coordinate, coincides with the instanton, i.e., the path with the highest tunneling probability [3-5]. If we denote the imaginary mode by $x$, a double-minimum potential $V(x)$, which connects the minima, is then a good zero-order approximation for the multidimensional potential governing the tunneling. To include the remaining normal modes $y_{i}$, we start from the

\footnotetext{
*Author to whom correspondence should be addressed: qf.ramos@usc.es

${ }^{\dagger}$ Permanent address: National Research Council of Canada, Ottawa, Canada, K1A 0R6.
}

transition state $x=0$ and include coupling terms $x^{m} y_{i}^{n}$ to describe these modes at other configurations. In practice the exponents $m, n$ can be restricted to the lowest value compatible with the symmetry of the system, specifically, to $n=1$. This procedure leads to a multidimensional imaginary-mode Hamiltonian (iMDH for short) whose accuracy peaks in the tunneling region rather than in the equilibrium configuration.

We have obtained satisfactory results by generating the iMDH from electronic-structure and force-field data of only two configurations: the stable configuration (EQ) and the transition state (TS), and evaluating the splittings by quasiclassical instanton techniques. Details and references to earlier work can be found in our recent papers [1]. A complicating factor in these calculations is that modes coupled to the tunneling require different handling depending on whether they move fast or slow compared to the tunneling mode, whose time constant is governed by the imaginary frequency. To a first approximation fast modes can be treated as "relaxed" and slow modes as "frozen," but some modes may fall into an intermediate region and cause ambiguity. To deal with this problem, we therefore propose here an alternative to the instanton approach, namely, a quantum method based on direct diagonalization of iMDH in a form with reduced dimensionality.

The Hamiltonian to be used in these calculations is basically the same as that used in the instanton calculations. The tunneling potential $V(x)$ and the couplings of the form $x y_{i}$ and $x^{2} y_{i}$ are derived as before [1,6]; in Sec. II we briefly review the form and properties of the iMDH. To reduce the dimensionality, a requirement to make the diagonalization tractable, we treat fast modes in the adiabatic approximation $[1,5]$. This makes the effective mass of the tunneling mode coordinate dependent, which poses a problem since a variable mass $m=$ $m(x)$ does not commute with the momentum operator $\hat{p}=$ $-i \hbar \partial / \partial x$ and the kinetic energy operator $\mathcal{T}[m(x)]$ is generally non-Hermitian. The problem has been amply discussed in the literature: Schrödinger equations with position-dependent mass have been studied for semiconductors [7], helium clusters [8], and superlattice band structures [9]. With respect to tunneling in molecular systems, this approach was applied 
to the inversion problem of the ammonia molecule $[10,11]$. However, these approaches are limited to one-dimensional (1D) systems. In Sec. III, we extend the position-dependent mass approach to the iMDH and apply it to evaluate tunneling splittings in real (multidimensional) systems where such splittings are observed. It has been asserted by von Roos [12] that the general Hermitian form of the kinetic energy operator $\mathcal{T}[m(x)]$ is not unique and that consequently, the effective mass approach is not suitable. However, it has been shown that there is a unique form of this operator which is compatible with the additional conditions of Galilean invariance and probabilistic interpretation of the wave function [13-15], and this is the form adopted in the present study.

In Sec. IV the approach is applied to the evaluation of the tunneling splitting of the zero-point level and the fundamentals of two symmetric modes in malonaldehyde and its monodeuterio isotopomer, a molecule with 18 vibrational degrees of freedom. The results are compared with those of other calculations, including our own, and with experiment. We briefly mention a second application, to the molecule porphycene, for which tunneling splittings have been recently measured and for which our calculations, to be reported in a forthcoming paper, yield results of comparable quality.

\section{THE IMAGINARY-MODE HAMILTONIAN}

Formulated in terms of the (mass-weighted) normal modes $\left(x,\left\{y_{i}\right\}\right)$ of the TS, taken as origin, and restricted to the lowestorder coupling terms allowed by symmetry, the iMDH is of the following general form:

$$
\begin{aligned}
\mathcal{H}= & -\frac{\hbar^{2}}{2} \frac{\partial^{2}}{\partial x^{2}}-\frac{\hbar^{2}}{2} \sum_{i} \frac{\partial^{2}}{\partial y_{i}^{2}}+V(x, y) ; \\
V(x, y)= & V(x)+\frac{1}{2} \sum_{i} \omega_{i}^{2} y_{i}^{2} \\
& -x^{2} \sum_{s} C_{s} y_{s}-x \sum_{a} C_{a} y_{a},
\end{aligned}
$$

where $V(x)$ is the double-minimum potential that connects the two minima $x= \pm \Delta x$, and subscripts $s$ and $a$ indicate modes that are symmetric and antisymmetric with respect to reflection in the plane $x=0$, i.e., modes that are displaced between the stationary configurations, namely, the EQ and TS configuration. The $s$ and $a$ modes are treated as harmonic oscillators with constant frequency $\omega_{s, a}$, and since coupling is linear in their coordinates, the coupling constants are proportional to the displacements:

$$
C_{s}=\omega_{s}^{2} \Delta y_{s} / \Delta x^{2} ; \quad C_{a}=\omega_{a}^{2} \Delta y_{a} / \Delta x^{2} .
$$

Undisplaced modes do not contribute to the Hamiltonian in this approximation. At the TS the kinetic energy is diagonal by definition; in our approach it is taken diagonal throughout, which is strictly valid only for collinear transfer. $V(x)$ is generated with the modes $y_{i}$ frozen in their equilibrium position ("crude-adiabatic" potential); its height $V_{0}$ and halfwidth $\Delta x$ are used as scaling parameters for the energy and the coordinates. In practice we operate with the adiabatic potential $V_{\text {ad }}(x)$ obtained from Eq. (1) as the relaxed potential

$$
\partial V(x, y) / \partial y=0 \text {. }
$$

Although the Hamiltonian (1) does not explicitly contain higher-order coupling terms proportional to $x y_{i}^{2}, x^{2} y_{i}^{2}$, etc., we can partially include their effect by imposing the condition that the adiabatic barrier height, which takes the form

$$
V_{\mathrm{ad}}(0)=V_{0}-\frac{1}{2} \Delta x^{4} \sum_{i} C_{i}^{2} / \omega_{i}^{2},
$$

equals the value computed quantum chemically for the real potential energy surface (PES). This leads to a rescaling of the coupling parameters $C_{i}$ in terms of the computed values $V_{0}, V_{\text {ad }}(0), \Delta x$, and $\omega_{i}$, as detailed in Ref. [1(a)]. To complete the description, one needs a function that describes $V_{\mathrm{ad}}(x)$ in the region $0<|x|<\Delta x$. This can be done by interpolating between the EQ and TS, where the curvatures $\partial^{2} V_{\text {ad }} / \partial x^{2}$ are known, or by using an adequate analytical function, e.g., a simple quartic function of the type

$$
V_{\mathrm{ad}}(x)=V_{\mathrm{ad}}(0)\left[1-(x / \Delta x)^{2}\right]^{2},
$$

found satisfactory for proton transfer in hydrogen bonds [1]. Formulated in terms of the adiabatic potential and with rescaled parameters, the $\mathrm{iMDH}$ used here thus takes the final form

$$
\begin{aligned}
\mathcal{H}= & -\frac{\hbar^{2}}{2} \frac{\partial^{2}}{\partial x^{2}}-\frac{\hbar^{2}}{2} \sum_{i} \frac{\partial^{2}}{\partial y_{i}^{2}}+V_{\mathrm{ad}}(x) \\
& +\frac{1}{2} \sum_{s} \omega_{s}^{2}\left(y_{s}-C_{s} x^{2} / \omega_{s}^{2}\right)^{2} \\
& +\frac{1}{2} \sum_{a} \omega_{a}^{2}\left(y_{a}-C_{a} x / \omega_{a}^{2}\right)^{2} .
\end{aligned}
$$

As an illustration, we derive in the Appendix the imaginarymode Hamiltonian [two-dimensional (2D) in this case] for the collinear exchange $\mathrm{AB}+\mathrm{C} \rightarrow \mathrm{A}+\mathrm{BC}$ described by the wellknown model Hamiltonian of two coupled Morse oscillators [16]. We solve numerically the 2D PES for the stationary configurations EQ and TS, define the normal modes of the TS, and generate all the relevant parameters. The result is a $2 \mathrm{D}$ analog of Eq. (6), where an adiabatic potential of the quartic type (5) is coupled to a single symmetric mode corresponding to the relative motion of the donor and acceptor "atoms," which is in excellent agreement with the original 2D potential, as seen from Fig. 1.

For a multidimensional (MD) system, the iMDH of the general form of Eq. (6) is generated from numerical electronicstructure and force-field data. The key point is that again only two configurations are needed: the EQ and the TS. Note also that it has a reduced dimensionality, since of the $3 \mathrm{~N}-7$ vibrational degrees of freedom $y_{i}$ of a nonlinear $N$-atomic system, only those contribute that are displaced between the stationary configurations.

\section{TUNNELING OF A COORDINATE-DEPENDENT MASS AND HERMITICITY}

As shown in our earlier work [1], there are at least four skeletal modes coupled to tunneling, even in the simple molecules for which experimental data are available, so that direct diagonalization would be unwieldy and the results would 

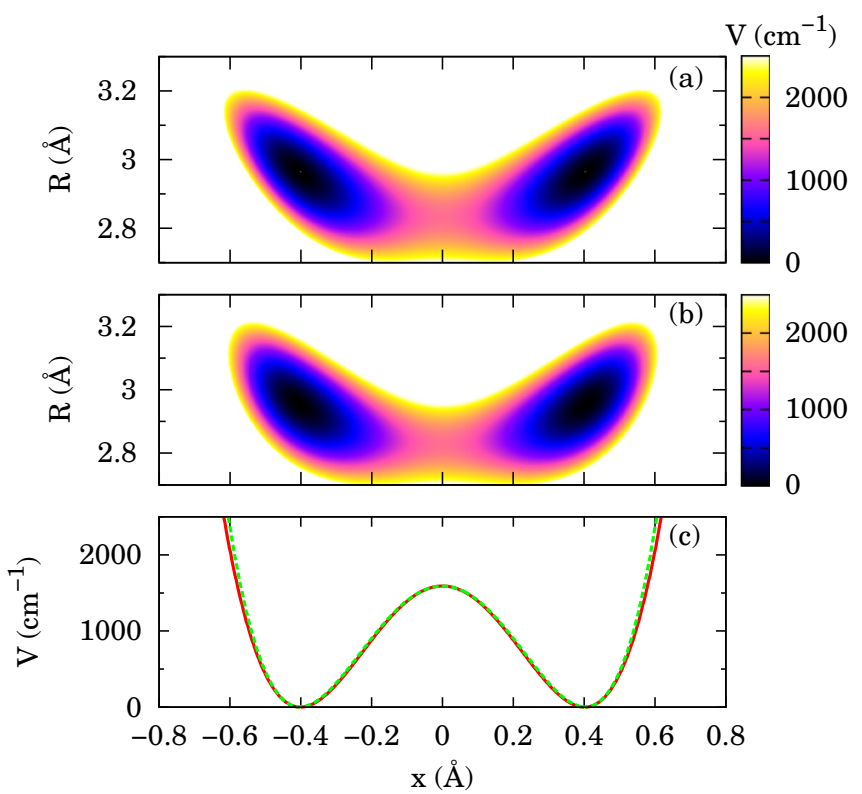

FIG. 1. (Color online) Contour energy plots comparing the 2D Morse potential (a) with the imaginary-mode Hamiltonian potential (b) for the collinear exchange reaction $\mathrm{B}+\mathrm{C} \rightarrow \mathrm{A}+\mathrm{BC}$ analyzed in the Appendix. Panel (c) plots the minimum energy path for the Morse potential (solid line) and for the imaginary-mode Hamiltonian (dashed line).

be difficult to interpret. To make this a viable method, it will be necessary to reduce the dimensionality by eliminating certain modes and treating them in an approximate way by rescaling parameters. This is possible under two conditions: their coupling to the tunneling must be relatively weak and they must be fast on the time scale of tunneling $t^{*}$ in $V_{\mathrm{ad}}(x)$, i.e., $\zeta_{i} \gg 1$, where $\zeta_{i}=\omega_{i} t^{*}$ (called zeta factors). In the next section we show that such modes can indeed be found for the systems considered, where normally the 1-2 strongest coupled modes are on the same time scale as tunneling; the remaining modes (marked by primes) are fast and their coupling is weak. In the instanton formalism such modes are treated in the adiabatic (or "slow flip") approximation [4]. This is equivalent to letting the potential in Eqs. (1) and (6) "relax" over such modes, leading to $y_{a^{\prime}}=\left[C_{a^{\prime}} / \omega_{a^{\prime}}^{2}\right] x ; \dot{y}_{a^{\prime}}=$ $\left[C_{a^{\prime}} / \omega_{a^{\prime}}^{2}\right] \dot{x}$ and $y_{s^{\prime}}=\left[C_{s^{\prime}} / \omega_{s^{\prime}}^{2}\right] x^{2} ; \dot{y}_{s^{\prime}}=2 x\left[C_{s^{\prime}} / \omega_{s^{\prime}}^{2}\right] \dot{x}$, which thus "eliminates" them from the dynamics but introduces a renormalized coordinate-dependent mass in the kinetic energy operator in the form $\mathcal{T}_{m}=\frac{1}{2} m(x) \dot{x}^{2}$ :

$m(x)=1+a+b x^{2} ; \quad a=\sum_{a^{\prime}} C_{a^{\prime}}^{2} / \omega_{a^{\prime}}^{4} ; \quad b=4 \sum_{s^{\prime}} C_{s^{\prime}}^{2} / \omega_{s^{\prime}}^{4}$.

This would appear to be an efficient way of reducing the dimensionality of the iMDH, but the mass renormalization poses a problem since the momentum operator $\hat{p}=-i \hbar \partial / \partial x$ does not commute with $m(x)$. It is therefore not immediately clear how to quantize the quasiclassical kinetic energy, as, for instance, the intuitive forms

$$
\mathcal{T}_{m}=\frac{1}{2 m(x)} \hat{p}^{2} ; \quad \mathcal{T}_{m}=\hat{p}^{2} \frac{1}{2 m(x)}
$$

are altogether non-Hermitian, which in general may give rise to unphysical results.

Although there is no unique way to define a Hermitian $\mathcal{T}_{m}[m(x)]$, the following two-parameter family known from the von Roos Hamiltonian [12] is often used as a general form:

$\mathcal{T}_{m}=\frac{1}{4}\left(m^{\alpha} \hat{p} m^{\beta} \hat{p} m^{\gamma}+m^{\gamma} \hat{p} m^{\beta} \hat{p} m^{\alpha}\right) ; \quad \alpha+\beta+\gamma=-1$,

where $m \equiv m(x)$. If this operator yields results that vary strongly for different combinations of the same parameters, we have a clear indication that the approach is unsound. For a given potential and coordinate-dependent mass, it will always be necessary to test the stability of the results. Such tests, carried out below, show that the quasiclassical kinetic energy $\mathcal{T}_{m}=\frac{1}{2} m(x) \dot{x}^{2}$ is compatible with Eq. (9), which yields stable (and very similar) eigenvalues. The tests also show that among all forms of the quantum kinetic energy operator (9), the lowest eigenvalues are obtained with the symmetric form

$$
\mathcal{T}_{m}=\hat{p} \frac{1}{2 m(x)} \hat{p},
$$

which involves $\alpha=\gamma=0$ and $\beta=-1$.

It has been shown that this monomial form is the only correct form of the kinetic energy operator with positiondependent mass. The additional conditions that make a unique choice possible are obtained from the general requirements of the probabilistic interpretation of the wave function and Galilean invariance [13-15]. Therefore in the applications of Sec. IV we adopt this form of the operator, $\mathcal{T}_{m}[m(x)]$.

In systems of practical interest, the coupling to tunneling in the Hamiltonians $(1,6)$ is generally dominated by symmetric modes, in particular, the stretching mode of the hydrogen bridge in proton-transfer reactions [1]; the usually much weaker coupling to antisymmetric modes can be handled by minor rescaling of parameters. Therefore we focus here on symmetric coupling. The Hamiltonian used in the diagonalization thus takes the final form

$$
\begin{aligned}
\mathcal{H}= & \hat{p} \frac{1}{2 m(x)} \hat{p}-\frac{\hbar^{2}}{2} \sum_{s \neq s^{\prime}} \frac{\partial^{2}}{\partial y_{s}^{2}}+V_{\mathrm{ad}}(x) \\
& +\frac{1}{2} \sum_{s \neq s^{\prime}} \omega_{s}^{2}\left(y_{s}-C_{s} x^{2} / \omega^{2}\right)^{2} .
\end{aligned}
$$

To our knowledge Eq. (11) represents the first multidimensional Hamiltonian with position-dependent mass, developed for the investigation of tunneling dynamics in molecular systems. One-dimensional tunneling with a coordinate-dependent mass was applied to the inversion vibration of the ammonia molecule $[10,11]$, based on a kinetic energy operator in Eq. (8), first form, in which case good agreement with experiment was obtained. In our case, the problem is more complicated since the tunneling mode couples strongly to several other vibrations. To handle the large number of basis functions required to achieve convergence of a kinetic energy operator of the form (10), we adopt an algorithm based on the JacobiDavidson method coded in the JADAMILU software [17], which is suitable for the calculation of selected eigenvalues and eigenvectors of large sparse matrices. As basis functions for 
TABLE I. Test of the JADAMILU algorithm for a 1D Hamiltonian of the form of Eq. (12). Column 2 lists the harmonic oscillator eigenvalues for unit mass and columns 3-5 those for a mass varying as $1+0.1 x^{2}$, calculated by different methods.

\begin{tabular}{ccccc}
\hline \hline $\mathrm{v}$ & $E_{v}^{0}$ & $E_{v}^{\mathrm{a}}$ & $E_{v}^{\mathrm{b}}$ & $E_{v}^{\mathrm{c}}$ \\
\hline 0 & 0.50000000 & 0.46889651 & 0.46889651 & 0.46889651 \\
1 & 1.50000000 & 1.43348555 & 1.43348555 & 1.43348555 \\
2 & 2.50000000 & 2.35654908 & 2.35654908 & 2.35654908 \\
3 & 3.50000000 & $3.24598(255)$ & 3.24598315 & 3.24598316 \\
4 & 4.50000000 & $4.10694(346)$ & 4.10694598 & 4.10694651 \\
5 & 5.50000000 & $4.943(37909)$ & 4.94320252 & 4.94320433 \\
\hline \hline
\end{tabular}

${ }^{a}$ Reference [18].

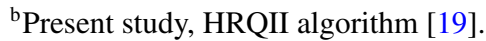

${ }^{\mathrm{c}}$ Present study, JADAMILU algorithm [17].

the tunneling we use harmonic-oscillator functions centered at $x=0$ with a zero-point amplitude $\Delta x$, which proved to be the most satisfactory approach.

Before application to MD systems, we tested the approach on 1D Hamiltonians with coordinate-dependent mass. As a first test we consider the 1D Hamiltonian

$$
\mathcal{H}_{1}=\hat{p} \frac{1}{2 m(x)} \hat{p}+\frac{1}{2} x^{2} ; \quad m(x)=1+0.1 x^{2},
$$

which is known from the literature [18]. We test JADAMILU against the standard diagonalization routine [19] (hereafter referred as HQRII), as well as against the method of Killenbeck [18]. The results, summarized in Table I, show that the JADAMILUalgorithm performs very well.

In the second test we study the adequacy of various forms of the kinetic energy operator in Eq. (9) (including the symmetric form (10), which is the correct one) in combination with a double-minimum potential of the quartic form, represented by Eq. (5):

$$
\begin{aligned}
\mathcal{H}_{2} & =\mathcal{T}_{m}[m(x)]+V_{0}\left[1-(x / \Delta x)^{2}\right]^{2} ; \\
m(x) & =1+\Delta m(x / \Delta x)^{2},
\end{aligned}
$$

for typical parameter values. The results in Table II for three forms (all Hermitian) show stable eigenvalues and virtually the same zero-point splittings, although the lowest eigenvalues are obtained with the symmetric form (10). This stability of the spectrum within the two-parameter family (9) justifies the use of a quantized form of $\mathcal{T}_{m}[m(x)]$ for the reduction of the dimensionality of the Hamiltonian calculation, so that only modes that are strongly coupled and have time constants similar to the tunneling mode need to be included in the diagonalization. The resulting Hamiltonian with the correct form (10) can then be used to calculate tunneling splittings, which are the observables of interest here. As an application of the Hamiltonian (11), we use this form of the generalized kinetic energy operator in the example of the next section.

\section{APPLICATIONS}

We now apply Hamiltonian (11) to calculate tunneling splittings in malonaldehyde, for which accurate experimental values have been reported for several vibrational levels [20-22]. The PES we take from recent quantum-chemical
TABLE II. Test for the Hamiltonian of the form of Eq. (13) with different forms of the Hermitian kinetic energy operator $\mathcal{T}_{m}[m(x)]$ given by Eqs. (9) and (10). The parameters are chosen so that the effect of mass renormalization is comparable with that in Table I: a quartic potential with barrier height $V_{0}=4232.74 \mathrm{~cm}^{-1}$, half-width $0.430 \AA \AA^{1 / 2}$, and frequency at the minimum of $2484.86 \mathrm{~cm}^{-1}$. Row 1 lists the first four eigenvalues for unit mass, interpreted as split pairs $E_{v}^{ \pm}$. Row 2 lists the splittings $\Delta_{v}$; all values are scaled by the frequency at the minimum. The same pattern is repeated for succeeding pairs of rows corresponding to a same mass of the form $1+0.328 x^{2}$ with the specified form of the kinetic energy operator $\mathcal{T}_{m}$. The results are obtained with the JADAMILU algorithm [17], which agrees with those obtained by the HRQII routine [19].

\begin{tabular}{lcccc}
\hline \hline & $0^{+}$ & $0^{-}$ & $1^{+}$ & $1^{-}$ \\
\hline$E_{v}^{0}$ & 0.47934 & 0.48017 & 1.31196 & 1.35761 \\
$\Delta_{v}$ & & 0.00083 & & 0.04565 \\
$E_{v}^{\mathrm{a}}$ & 0.41836 & 0.41883 & 1.18316 & 1.20631 \\
$\Delta_{v}$ & & 0.00048 & & 0.02315 \\
$E_{v}^{\mathrm{b}}$ & 0.42083 & 0.42129 & 1.18777 & 1.21031 \\
$\Delta_{v}$ & & 0.00046 & & 0.02254 \\
$E_{v}^{\mathrm{c}}$ & 0.41926 & 0.41972 & 1.18655 & 1.20901 \\
$\Delta_{v}$ & & 0.00046 & & 0.02246 \\
\hline
\end{tabular}

${ }^{\mathrm{a}} \mathcal{T}_{m}$ as in Eq. (10) [same as Eq. (9), for $\alpha=\gamma=0, \beta=-1$ ].

${ }^{\mathrm{b}} \mathcal{T}_{m}$ as in Eq. (9), $\alpha=\gamma=-0.5, \beta=0$.

${ }^{\mathrm{c}} \mathcal{T}_{m}$ as in Eq. (9), $\alpha=-1, \beta=\gamma=0$.

results calculated at the EQ and the TS [1a]. The relevant calculated parameters of the iMDH are listed in Table III. From these data we calculate the parameters that enter the Hamiltonian to be diagonalized, which are also listed.

Although so far we have discussed only symmetric modes, we now include antisymmetric modes as well. Since they are weakly coupled, this coupling can be treated approximately and as independent from the symmetric coupling [23]. Namely, we divide these modes into fast and slow, and treat modes with $\zeta_{i}>1$ and $\zeta_{i}<1$ in the adiabatic ("slow flip") and sudden ("fast flip") approximation, respectively [4,23]. Fast modes

TABLE III. Calculated parameters of the imaginary-mode Hamiltonian (6) for malonaldehyde- $d_{0}$ and $-d_{1}$, listed as $\left(d_{0}\right) /\left(d_{1}\right),\left(d_{0}\right) /-$ (parameter just for malonaldehyde- $d_{0}$ ) and $-/\left(d_{1}\right)$ (parameter just for malonaldehyde- $\left.d_{1}\right)$. All parameters are taken from Tables II and III of Ref. [1(a)]. Frequencies $\omega$ are in $\mathrm{cm}^{-1}$, the other parameters viz. coupling parameters $\gamma_{i}$ and $B_{i}$ and zeta factors $\zeta_{i}$ from Eq. (14) are dimensionless. The barrier heights are 4.08 (adiabatic) and 14.44 (crude-adiabatic) $\mathrm{kcal} / \mathrm{mol}$, and the barrier half-width equals 0.430 Åamu ${ }^{1 / 2}$; the scaling frequency equals $\Omega=960 \mathrm{~cm}^{-1}$.

\begin{tabular}{lcccc}
\hline \hline$i$ & $\omega_{i}$ & $\gamma_{i}$ & $B_{i}$ & $\zeta_{i}$ \\
\hline$s=1$ & $627 / 627$ & $0.64 / 0.85$ & $0.48 / 0.47$ & $1.2 / 1.6$ \\
$s=2$ & $943 / 930$ & $0.38 / 0.59$ & $0.07 / 0.10$ & $1.9 / 2.4$ \\
$s$ & $1361 / 1340$ & $0.24 / 0.81$ & $0.01 / 0.09$ & $2.2 / 3.5$ \\
$s$ & $1896 /-$ & $1.01 /-$ & $0.13 /-$ & $3.7 /-$ \\
$s$ & $-/ 1387$ & $-/ 0.46$ & $-/ 0.03$ & $-/ 3.6$ \\
$s$ & $-/ 1635$ & $-/ 0.24$ & $-/ 0.01$ & $-/ 4.3$ \\
$a$ & $1604 /-$ & $0.25 /-$ & $0.01 /-$ & $3.1 /-$ \\
$a$ & $-/ 568$ & $-/ 0.09$ & $-/ 0.01$ & $-/ 1.5$ \\
\hline \hline
\end{tabular}


TABLE IV. Eigenvalues and tunneling splittings for malonaldehyde- $d_{0}$ (top) and $-d_{1}$ (bottom) calculated for the parameters listed in Table III. A dash indicates that the experimental value is not available.

\begin{tabular}{lcccccccc}
\hline \hline$E_{0}^{+}$ & $E_{0}^{-}$ & $\Delta_{0} / \Delta_{0}^{\mathrm{obs}}$ & $E_{1}^{+}$ & $E_{1}^{-}$ & $\Delta_{1} / \Delta_{1}^{\mathrm{obs}, \mathrm{c}}$ & $E_{2}^{+}$ & $E_{2}^{-}$ & $\Delta_{2} / \Delta_{2}^{\mathrm{obs}, \mathrm{c}}$ \\
\hline 1637.9 & 1663.3 & $25.4 / 21.58^{\mathrm{a}}$ & 1923.5 & 2015.9 & $92.4 / 57$ & 2540.9 & 2572.5 \\
1449.8 & 1453.0 & $3.2 / 2.92^{\mathrm{b}}$ & 1764.1 & 1788.4 & $24.3 /-$ & 2326.6 & 2333.5 & $6.9 / 27$ \\
\hline \hline
\end{tabular}

${ }^{\mathrm{a}}$ Reference [20].

${ }^{\mathrm{b}}$ Reference [21].

${ }^{\mathrm{c}}$ Reference [22].

yield (minor) correction of the adiabatic barrier height and constant (also minor) increase of the tunneling mass; slow modes contribute a Franck-Condon factor to the calculated splitting [23]. All these effects are negligible here [1a].

To deal with the coupled symmetric modes, we first single out the two modes with the strongest coupling for direct diagonalization, which also are the two modes with $\zeta \sim 1$. While the limitation to two modes is made in part for practical reasons, it turns out to be a logical choice for hydrogenbonded systems in which the coupling is always dominated by the hydrogen-bond stretching mode. The coupled symmetric modes not included in the direct diagonalization are all fast and are treated collectively in the adiabatic approximation. They contribute a term of the form $b x^{2}$ to the mass renormalization. It is convenient to use dimensionless parameters by expressing energies in units $V_{0}$, coordinates in units $\Delta x$, and frequencies and time in units of the scaling frequency $\Omega$, defined as $\Omega^{2} \Delta x^{2}=V_{0}$ [1a]. This leads to a 3D Hamiltonian of the form

$$
\begin{aligned}
\mathcal{H}_{3 \mathrm{D}} & =\mathcal{H}_{1 \mathrm{D}}(x)+\sum_{s=1,2} \mathcal{H}_{s}\left(y_{s}\right) \\
\mathcal{H}_{1 \mathrm{D}} & =-g^{2} \frac{\partial}{\partial x} \frac{1}{m(x)} \frac{\partial}{\partial x}+(1-B)\left(1-x^{2}\right)^{2} ; \\
\mathcal{H}_{s}\left(y_{s}\right) & =-g^{2} \frac{\partial^{2}}{\partial y_{s}^{2}}+\frac{1}{2} \omega_{s}^{2}\left(y_{s}-\frac{\gamma_{s}}{\omega_{s}^{2}} x^{2}\right)^{2}
\end{aligned}
$$

where $g^{2}=\hbar^{2} / 2 V_{0} \Delta x^{2}, \quad \gamma_{s}=C_{s} / \Delta x^{2}, \quad B=\sum_{i} B_{i}=$ $\sum_{i} \gamma_{i} / 2 \omega_{i}^{2}$, and $m(x)=1+\Delta m x^{2}$ with $\Delta m=4 \sum_{s^{\prime}} \gamma_{s^{\prime}}^{2} / \omega_{s^{\prime}}^{4}$, the sum running over all but the first two modes directly included in the above Hamiltonian. In these units the characteristic time of motion in the adiabatic potential is $1 / \sqrt{1-B}$, so that the parameters $\zeta_{i}$ used to classify the modes are given by $\zeta_{i}=\omega_{i} / \sqrt{1-B}$.

To generate the matrix for diagonalization we use as a basis set the combination

$$
\psi\left(x, y_{1}, y_{2}\right)=\sum_{m, n, k} C_{m, n, k} \phi_{m}(x) \phi_{n}\left(y_{1}\right) \phi_{k}\left(y_{2}\right),
$$

where the functions $\phi$ are the eigenfunctions of the zero-order Hamiltonians,

$$
\mathcal{H}_{0}=g^{2}\left(-\frac{\partial^{2}}{\partial x^{2}}+x^{2}\right) ; \quad \mathcal{H}_{s}^{0}\left(y_{s}\right)=-g^{2} \frac{\partial^{2}}{\partial y_{s}^{2}}+\frac{1}{2} \omega_{s}^{2} y_{s}^{2},
$$

respectively. While the matrix elements of the potential are straightforward, those of the kinetic energy operator tend to require a large number of functions $\phi_{m}$ before convergence is achieved, since there are no clear selection rules. For this particular case the order of the matrix is $200 \times(15)^{2}$. Nevertheless, the matrix generated over the basis (15) is rather sparse and is therefore amenable to the JADAMILU algorithm tested in the preceding section.

As a demonstration we apply the method to proton tunneling in two isotopomers of malonaldehyde, $\mathrm{O}=\mathrm{CH}$ $\mathrm{CH}=\mathrm{CH}-\mathrm{OH}$ and $\mathrm{O}=\mathrm{CH}-\mathrm{CH}=\mathrm{CH}-\mathrm{OD}$. Of the 18 normal modes at the TS, eight are symmetric and six antisymmetric; the remaining four are neither and therefore do not contribute to the iMDH in Eqs. (1) and (6). The input parameters are listed in Table III; they are based on the same quantumchemical calculations as those used for the same purpose but with the rainbow instanton approximation in Ref. [1(a)]. In Table IV the calculated splittings are compared with the available experimental data. Although they are somewhat larger than the observed splittings [20-22], they account well for the observed deuterium isotope effect and the effect of vibrational excitation [21]. The zero-point splittings are virtually indistinguishable from those previously calculated with the rainbow instanton method [1a], which indicates that their accuracy is limited by the calculated potential and not by the dynamics used in either calculation. The splittings calculated for the fundamentals agree well with the much more elaborate calculations of Hammer and Manthe [24].

To illustrate the nature of the coupling effects, we also carried out calculations with a 2D version of the Hamiltonian in Eq. (14), where tunneling is coupled to the symmetric stretching of the hydrogen bridge (mode $y_{1}$ ). Figure 2 plots the effect of coupling on the probability density of the $0^{+}$state when $x$ and $y_{1}$ are uncoupled (solid line), which corresponds to tunneling in the "partially adiabatic" potential $\left(1-B^{\prime}\right)\left(1-x^{2}\right)^{2}, B^{\prime}=\sum_{s^{\prime}} B_{s^{\prime}}$, with barrier height reduced by the fast modes but no mass effect, plus the harmonic oscillator. The dashed line represents this probability density function when $x$ and $y_{1}$ are still uncoupled but the mass effect of the fast modes is included; thus the solid and dashed lines illustrate the effect of the fast modes. Finally, the dotted line represents the combined effect, with coupling to $y_{1}$ included. The figure shows that slow and fast symmetric modes affect the spread of the wave function and thus the magnitude of the tunneling splitting in opposite directions.

Malonaldehyde is commonly used as a benchmark singleproton transfer system to test new models that evaluate tunneling splittings, but the method described in this paper is robust enough to deal with multiple-proton transfer in complex molecules. To probe this, we have investigated the molecule porphycene, a molecule of biological interest that presents a case of maximum complexity: a potentialenergy surface with nine stationary points, two tunneling 


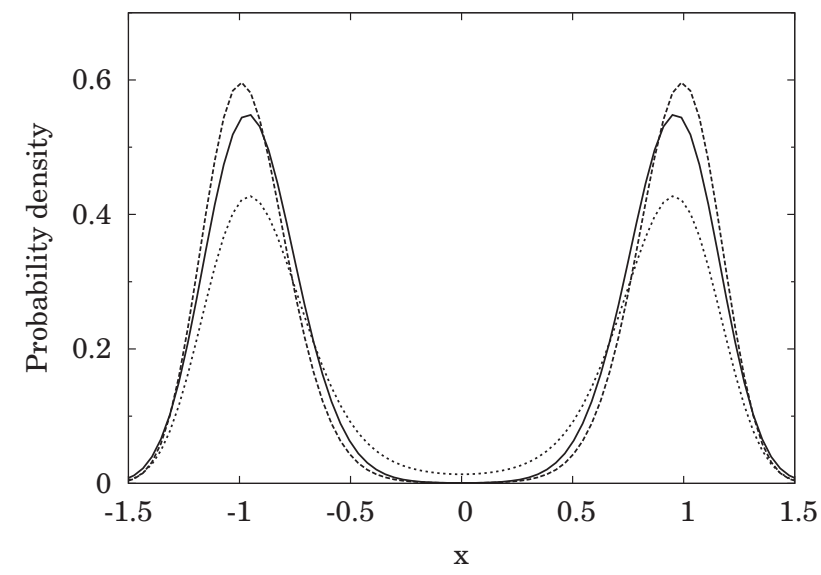

FIG. 2. Illustration of the coupling effects described by a $2 \mathrm{D}$ version of Hamiltonian (14) for malonaldehyde- $d_{0}$, which describes tunneling with renormalized mass coupled to the symmetric stretching of the hydrogen bridge (mode $y_{1}$ ). Plot of the maximum probability density $\left|\Psi_{0}^{(+)}\left(x, y_{1}\right)\right|^{2}$ when $y_{1}$ is varied versus the tunneling coordinate $x$ (dimensionless). See text for details.

coordinates, and antisymmetric as well as symmetric coupled modes. The Hamiltonian to be diagonalized in this case is a generalization to that in Eq. (14) and corresponds to tunneling with coordinate-dependent mass coupled to one antisymmetric mode and three symmetric modes. Detailed calculations, to be reported in a forthcoming paper [25], yield results that compare as well with recently measured tunneling splittings [26-28] as those for malonaldehyde. A zero-point splitting of $4.1 \mathrm{~cm}^{-1}$ and a splitting of the fundamental of the hydrogen-bridge mode of $10.3 \mathrm{~cm}^{-1}$ compared to observed values of 4.43 and $12 \mathrm{~cm}^{-1}$, respectively.

\section{CONCLUSION}

On the basis of these results, we conclude that the imaginary-mode Hamiltonian presented here is a sound and efficient approach to study tunneling dynamics in MD systems. It is very economical, since the generation of the $\mathrm{iMDH}$ in Eq. (6) requires standard electronic-structure data and the Hessians of only two configurations: the stable configuration and the transition state. The Hamiltonian with reduced dimensionality in Eq. (11), obtained by this $\mathrm{MDHH}$, represents tunneling with a coordinate-dependent mass, representing fast modes, coupled to those degrees of freedom whose time scale is similar to that of the tunneling motion. Test results show that 1D Hamiltonians of this type have stable eigenvalues for the chosen form of the Hermitian kinetic energy operator. The strength of the coordinate dependence of the mass is calculated from the displacements of the tunneling mode and the fast modes between the equilibrium position and the transition state. The matrix elements of the Hamiltonian, with up to two strongly coupled modes in terms of a basis set of harmonic oscillator functions centered at the transition state, lead to a sparse matrix which can be handled by the JADAMILU algorithm to yield values for the tunneling splitting of the zero-point level and the fundamental levels of the coupled modes. Application to two isotopomers of malonaldehyde, a molecule that is frequently used as a benchmark for these properties, confirms that even for the simplified potentials used here, based on only two calculated configurations, the iMDH can give a satisfactory account of these tunneling splittings and their isotope effect.

\section{ACKNOWLEDGMENTS}

We are grateful to an anonymous reviewer for drawing our attention to Refs. [13] and [14].

\section{APPENDIX}

Here we derive the imaginary-mode Hamiltonian of Eq. (6) (i2DH in this case) for the collinear exchange $\mathrm{AB}+\mathrm{C} \rightarrow \mathrm{A}+$ $\mathrm{BC}$, described by the well-known model Hamiltonian of two coupled Morse oscillators [16]:

$$
\begin{aligned}
\mathcal{H}_{2 \mathrm{D}}= & -\frac{\hbar^{2}}{2}\left[\left(\frac{1}{m}+\frac{1}{M}\right)\left(\frac{\partial^{2}}{\partial r_{\mathrm{AB}}^{2}}+\frac{\partial^{2}}{\partial r_{\mathrm{BC}}^{2}}\right)-\frac{2}{m} \frac{\partial}{\partial r_{\mathrm{AB}}} \frac{\partial}{\partial r_{\mathrm{BC}}}\right] \\
& +D\left[e^{-\beta\left(r_{\mathrm{AB}}-r_{0}\right)}-1\right]^{2}+D\left[e^{-\beta\left(r_{\mathrm{BC}}-r_{0}\right)}-1\right]^{2} \\
& +\frac{1}{2} K\left(R-R_{0}\right)^{2},
\end{aligned}
$$

where $M=m_{\mathrm{A}}=m_{\mathrm{C}}, m=m_{\mathrm{B}}, R \equiv r_{\mathrm{CA}}=r_{\mathrm{C}}-r_{\mathrm{A}}$, and $r_{0}$ and $R_{0}$ denote the equilibrium values of the corresponding bond lengths. Introducing $X=r_{\mathrm{B}}-\left(r_{\mathrm{C}}+r_{\mathrm{A}}\right) / 2$ and excluding the center of mass, Eq. (A1) takes the form

$$
\begin{aligned}
\mathcal{H}_{2 \mathrm{D}}= & -\frac{\hbar^{2}}{2 \mu_{0}} \frac{\partial^{2}}{\partial X^{2}}-\frac{\hbar^{2}}{2 \mu} \frac{\partial^{2}}{\partial R^{2}} \\
& +D\left[e^{-\beta\left(R / 2-X-r_{0}\right)}-1\right]^{2}+D\left[e^{-\beta\left(R / 2+X-r_{0}\right)}-1\right]^{2} \\
& +\frac{1}{2} K\left(R-R_{0}\right)^{2},
\end{aligned}
$$

where $\mu_{0}=2 M m /(2 M+m)$ and $\mu=M / 2$. The 2 D potential in Eq. (A2) is illustrated in Fig. 1 for a set of parameters chosen so as to mimic the real parameters of proton transfer along the hydrogen bridge $\mathrm{O}-\mathrm{H} \cdots \mathrm{O} \rightarrow \mathrm{O} \cdots \mathrm{H}-\mathrm{O}$ in malonaldehyde, treated in Sec. IV, namely, $M=20$ amu, $m=1 \mathrm{amu}, \beta=$ $2.1 \AA^{-1}, R_{0}=3.12 \AA, r_{0}=1 \AA, D=33715 \mathrm{~cm}^{-1}$, and $K=120000 \mathrm{~cm}^{-1} \AA^{-2}$. It describes tunneling in a symmetric double-minimum potential, whose properties are modulated by the heavy "atoms" serving as proton donor and acceptor.

To generate the i2DH in the form of Eq. (6), we need the values $X$ and $R$ as well as $V(X, R)$ at the stationary configurations EQ and TS, used as subscripts, and the normal modes at the TS. Rewriting $V(x, y)$ in Eq. (6) as $V(X, R)$, we note that the crude-adiabatic potential equals $V\left(X ; R_{\mathrm{EQ}}\right)$. The conditions $\partial V(X, R) / \partial X=0, \partial V(X, R) / \partial R=0$ for stationary configurations yield, when solved numerically, the values of $X_{\mathrm{EQ}}, R_{\mathrm{EQ}}, X_{\mathrm{TS}}=0, R_{\mathrm{TS}}, V\left(X_{\mathrm{EQ}}, R_{\mathrm{EQ}}\right)$, and $V\left(0, R_{\mathrm{TS}}\right)$. Thus the adiabatic barrier half-width and height are $X_{\mathrm{EQ}}$ and $V_{\mathrm{ad}}(0)=V\left(0, R_{\mathrm{TS}}\right)-V\left(X_{\mathrm{EQ}}, R_{\mathrm{EQ}}\right)$, respectively. This defines the barrier height $V_{0}=V\left(0, R_{\mathrm{EQ}}\right)-$ $V\left(X_{\mathrm{EQ}}, R_{\mathrm{EQ}}\right)$, used in our approach, as the scaling parameter for energy. The (mass-weighted) normal modes $x, y$ at the $\mathrm{TS}$, found from $\left[\partial^{2} V(X, R) / \partial x \partial y\right]_{\mathrm{TS}}=0$, are $x=\sqrt{\mu_{0}} X$ and $y=\sqrt{\mu}\left(R-R_{\mathrm{TS}}\right)$, with the corresponding squared frequencies $\left|\omega^{*}\right|^{2}=\left[\partial^{2} V(X, R) / \partial x^{2}\right]_{\mathrm{TS}}$ and $\omega^{2}=\partial^{2} V(X, R) / \partial y^{2}$; since these modes are taken to be zero at the TS, their 
displacements in the EQ assume the values $\Delta x=\sqrt{\mu_{0}} X_{\mathrm{EQ}}$ and $\Delta y=\sqrt{\mu}\left(R_{\mathrm{EQ}}-R_{\mathrm{TS}}\right)$. The above parameters of the original Hamiltonian (A1) yield the following values for the parameters of the 2D imaginary-mode Hamiltonian: $\mu_{0}=$ $0.976 \mathrm{amu}, \mu=10 \mathrm{amu}, X_{\mathrm{EQ}}=0.403 \AA, R_{\mathrm{EQ}}=2.966 \AA$, $R_{\mathrm{TS}}=2.833 \AA, V\left(X_{\mathrm{EQ}}, R_{\mathrm{EQ}}\right)=26269.6 \mathrm{~cm}^{-1}, V\left(0, R_{\mathrm{TS}}\right)=$ $28813.5 \mathrm{~cm}^{-1}, V_{\mathrm{ad}}(0)=1590.6 \mathrm{~cm}^{-1}, V_{0}=2543.9 \mathrm{~cm}^{-1}$, $\omega^{*}=1193 i \mathrm{~cm}^{-1}, \omega=636 \mathrm{~cm}^{-1}, \Delta x=0.398 \AA_{a m u}^{1 / 2}$, and $\Delta y=0.399 \AA^{\circ} \mathrm{amu}^{1 / 2}$. These parameters define the coupling constant [renormalized as in Eq. (4)]: $C=1018344.2$ $\mathrm{cm}^{-2} \AA^{-1} \mathrm{amu}^{-1 / 2}$. The adiabatic (relaxed) potential $V_{\mathrm{ad}}(x)$ is obtained from the condition $\partial V(X, R) / \partial R=0$, which defines $R_{\text {ad }}(x)$. Two conclusions follow from this numerical analysis: first, that $V_{\mathrm{ad}}(x)$ is very well approximated by the quartic function defined in Eq. (5); and second, that $R_{\mathrm{ad}}(x)$ is similarly represented by the analytical expression $R_{\mathrm{ad}}(x)=R_{\mathrm{TS}}+$ $\left(R_{\mathrm{EQ}}-R_{\mathrm{TS}}\right)(x / \Delta x)^{2}$. This immediately allows representation of $\mathcal{H}_{2 \mathrm{D}}-V\left(X_{\mathrm{EQ}}, R_{\mathrm{EQ}}\right)$ as a $2 \mathrm{D}$ imaginary-mode Hamiltonian in the form of Eq. (6), with a quartic potential coupled to a single symmetric mode:

$$
\begin{aligned}
\mathcal{H}_{\mathrm{i} 2 \mathrm{D}}= & -\frac{\hbar^{2}}{2} \frac{\partial^{2}}{\partial x^{2}}-\frac{\hbar^{2}}{2} \frac{\partial^{2}}{\partial y^{2}}+V_{\mathrm{ad}}(0)\left[1-(x / \Delta x)^{2}\right]^{2} \\
& +\frac{1}{2} \omega^{2}\left(y-C x^{2} / \omega^{2}\right)^{2} .
\end{aligned}
$$

The potentials of $\mathcal{H}_{\mathrm{i} 2 \mathrm{D}}$ and $\mathcal{H}_{2 \mathrm{D}}$ are superimposed in Fig. 1 to illustrate their similarity.
[1] Z. Smedarchina, W. Siebrand, and A. Fernández-Ramos, J. Chem. Phys. 137, 224105 (2012); J. Phys. Chem. A 117, 11086 (2013).

[2] Z. Smedarchina, W. Siebrand, M. Z. Zgierski, and F. Zerbetto, J. Chem. Phys. 102, 7024 (1995); Z. Smedarchina, W. Siebrand, and M. Z. Zgierski, ibid. 103, 5326 (1995).

[3] S. Coleman, in The Whys of Subnuclear Physics, edited by A. Zichichi (Plenum, New York, 1979), p. 805.

[4] A. I. Vainshtein, V. I. Zakharov, V. A. Novikov, and M. A. Shifman, Sov. Phys. Usp. 25, 195 (1982).

[5] J. P. Sethna, Phys. Rev. B 24, 698 (1981); 25, 5050 (1982).

[6] W. Siebrand, Z. Smedarchina, M. Z. Zgierski, and A. FernándezRamos, Int. Rev. Phys. Chem. 18, 5 (1999).

[7] G. Bastard, Wave Mechanics Applied to Semiconductor Heterostructures (Les Editions de Physique, Les Ulis, 1988).

[8] M. Barranco, M. Pi, S. M. Gatica, E. S. Hernandez, and J. Navarro, Phys. Rev. B 56, 8997 (1997).

[9] G. Bastard, Phys. Rev. B 24, 5693 (1981).

[10] N. Aquino, G. Campoy, and Yee-Madeira, Chem. Phys. Lett. 296, 111 (1998).

[11] J. Förster, A. Saenz, and U. Wolff, Phys. Rev. E 86, 016701 (2012).

[12] O. von Roos, Phys. Rev. B 27, 7547 (1983).

[13] P. V. Elyutin and V. D. Krivchenkov, Theor. Math. Phys. 16, 939 (1973).

[14] I. M. Sokolov, Z. Exp. Teor. Fiz. 88, 556 (1985) [Sov. Phys.JETP (USA) 62, 317 (1985)].

[15] J. Thomsen, G. T. Einevoll, and P. C. Hemmer, Phys. Rev. B 39, 12783 (1989).
[16] B. S. Butayev, A. S. Saakanyan, and V. P. Spiridonov, Chem. Phys. Lett. 138, 303 (1987); N. Sato and S. Iwata, J. Chem. Phys. 89, 2932 (1988).

[17] M. Bollhöfer and Y. Notay, Comput. Phys. Commun. 177, 951 (2007).

[18] J. P. Killingbeck, J. Phys. A: Math. Theor. 44, 285208 (2011); R. Koç and S. Sayın, ibid. 43, 455203 (2010).

[19] Y. Beppu and I. Nonomi, Comput. Chem. 6, 87 (1982).

[20] D. W. Firth, K. Beyer, M. A. Dvorak, S. W. Reeve, A. Q. Gushov, and K. Leopold, J. Chem. Phys. 94, 1812 (1991); T. Baba, T. Tanaka, I. Morino, K. M. T. Yamada, and K. Tanaka, ibid. 110, 4131 (1999).

[21] S. L. Baughcum, Z. Smith, E. B. Wilson, and R. W. Duerst, J. Am. Chem. Soc. 106, 2260 (1984).

[22] T. N. Wassermann, D. Luckhaus, S. Coissan, and M. A. Suhm, Phys. Chem. Chem. Phys. 8, 2344 (2006); N. O. B. Lüttschwager, T. N. Wassermann, S. Coissan, and M. A. Suhm, ibid. 12, 8201 (2010); Mol. Phys. 111, 2211 (2013).

[23] V. A. Benderskii and D. E. Makarov, Chem. Phys. 170, 275 (1993).

[24] T. Hammer and U. Manthe, J. Chem. Phys. 136, 054105 (2012).

[25] Z. Smedarchina, A. Fernández-Ramos, and W. Siebrand (unpublished).

[26] J. Sepioł, Y. Stepanenko, A. Vdovin, A. Mordziński, E. Vogel, and J. Waluk, Chem. Phys. Lett. 296, 549 (1998).

[27] J. Waluk, Acc. Chem. Res. 39, 945 (2006).

[28] A. Vdovin, J. Waluk, B. Dick, and A. Slenczka, ChemPhysChem 10, 761 (2009). 